\title{
Constrained quantization of open string in background B field and non-commutative D-brane
}

\author{
Chong-Sun Chu ${ }^{\text {a }}$, Pei-Ming Ho ${ }^{b}$ \\ ${ }^{\mathrm{a}}$ Institute of Physics, University of Neuchâtel, CH-2000 Neuchâtel, Switzerland \\ ${ }^{\mathrm{b}}$ Department of Physics, National Taiwan University, Taipei 10764, Taiwan, China
}

Received 8 July 1999; accepted 25 October 1999

\begin{abstract}
In a previous paper we provided a consistent quantization of open strings ending on D-branes with a background $B$ field. In this letter, we show that the same result can also be obtained using the more traditional method of Dirac's constrained quantization. We also extend the discussion to the fermionic sector. (C) 2000 Elsevier Science B.V. All rights reserved.
\end{abstract}

\section{Introduction}

It was shown by Connes, Douglas and Schwarz [1] that for the Matrix model compactified on a torus, the three-form $C$ field background should be incorporated in the supersymmetric Yang-Mills theory (SYM) by deforming the base space into a quantum space. Due to the correspondence between the DLCQ M-theory and the D-brane world-volume field theory [2], this means that the D-brane field theory lives on a non-commutative space in the presence of a NS-NS $B$ field background [3-5]. In a previous paper we quantized an open string ending on a D-brane with a constant NS-NS $B$ field background, and we found that the space-time coordinates of the open string endpoint are non-commutative. The non-commutativity precisely agrees with previous results [1], but in addition we showed that the compactness of the torus is not necessary for the non-commutativity, and in general the deformation is parametrized by $\mathscr{F}=B-F$ instead of $B$.

The quantization of an open string that we did in Ref. [6] was carried out explicitly in terms of a mode expansion of the generic solution of the equations of motion for the space-time coordinates $X^{\mu}$. We used a heuristic argument in the derivation of commutation relations among the mode coefficients, and then check that it is a consistent quantization of the classical theory.

E-mail addresses: cschu@sissa.it (C.-S. Chu), pmho@phys.ntu.edu.tw (P.-M. Ho). 
In this paper we carry out the quantization again by following the standard quantization procedure of Dirac for systems with constraints. The constraints here are, of course, the boundary conditions of the open string ending on a D-brane. We find that there is a single ambiguous expression which needs to be regularized. We obtain our previous results by choosing a natural regularization. We also find the commutation relations for the fermionic fields using supersymmetry.

\section{Necessity of non-commutativity}

We first review briefly the setting. Consider a fundamental string ending on a D p-brane. This can be in type IIsuperstring, type 0 superstring, or in the bosonic string theory. The bosonic part of the action takes the same form [7,8]

$$
\begin{aligned}
S_{B}= & \frac{1}{4 \pi \alpha^{\prime}} \int_{\Sigma} d^{2} \sigma\left[g^{\alpha \beta} G_{\mu \nu} \partial_{\alpha} X^{\mu} \partial_{\beta} X^{\nu}+\epsilon^{\alpha \beta} B_{\mu \nu} \partial_{\alpha} X^{\mu} \partial_{\beta} X^{\nu}\right] \\
& +\frac{1}{2 \pi \alpha^{\prime}} \oint_{\partial \Sigma} d \tau A_{i}(X) \partial_{\tau} X^{i}
\end{aligned}
$$

where $A_{i}, i=0,1, \ldots, p$, is the $U(1)$ gauge field living on the $\mathrm{D} p$-brane. We use the convention $\eta^{\alpha \beta}=\operatorname{diag}(-1,1)$ and $\epsilon^{01}=1$ as in [6]. Here the string background is

$$
G_{\mu \nu}=\eta_{\mu \nu}, \quad \Phi=\text { constant }, \quad H=d B=0 .
$$

Adding the fermionic sector does not affect the result and will be considered later. With slight modification, the considerations in this paper can also be applied to study open string ending on a D-brane in type I string theory.

If both ends of a string are attached to the same D p-brane, the last term in (1) can be written as

$$
\frac{-1}{4 \pi \alpha^{\prime}} \int_{\Sigma} d^{2} \sigma \epsilon^{\alpha \beta} F_{i j} \partial_{\alpha} X^{i} \partial_{\beta} X^{j}
$$

Furthermore, consider the case $B=\sum_{i, j=0}^{p} B_{i j} d X^{i} d X^{j}$, then the action (1) can be written as

$$
S_{B}=-\int d \tau L=\frac{1}{4 \pi \alpha^{\prime}} \int d^{2} \sigma\left[g^{\alpha \beta} \eta_{\mu \nu} \partial_{\alpha} X^{\mu} \partial_{\beta} X^{\nu}+\epsilon^{\alpha \beta} \mathscr{F}_{i j} \partial_{\alpha} X^{i} \partial_{\beta} X^{j}\right]
$$

Here

$$
\mathscr{F}=B-d A=B-F
$$

is the modified Born-Infeld field strength and $x_{0}^{a}$ is the location of the D-brane. Indices are raised and lowered by $\eta_{i j}=(-,+, \ldots,+)$.

One obtains the equations of motion

$$
\left(\partial_{\tau}^{2}-\partial_{\sigma}^{2}\right) X^{\mu}=0
$$

and the boundary conditions at $\sigma=0, \pi$ :

$$
\begin{aligned}
& \partial_{\sigma} X^{i}+\partial_{\tau} X^{j} \mathscr{F}_{j}{ }^{i}=0, \quad i, j=0,1, \ldots, p, \\
& X^{a}=x_{0}^{a}, \quad a=p+1, \ldots, D .
\end{aligned}
$$


The constraint (8) is standard. We will be mainly interested in the constraint (7). As demonstrated in [6], the BC (7) implies that

$$
2 \pi \alpha^{\prime} P^{k}(\tau, 0) \mathscr{F}_{k}^{i}=-\partial_{\sigma} X^{j}(\tau, 0) M_{j}^{i},
$$

where $P^{k}$ is the canonical momentum

$$
2 \pi \alpha^{\prime} P^{k}(\tau, \sigma)=\partial_{\tau} X^{k}+\partial_{\sigma} X^{j} \mathscr{F}_{j}^{k},
$$

and $M_{i j}=\eta_{i j}-\mathscr{F}_{i}{ }^{k} \mathscr{F}_{k j}$. It follows that

$$
2 \pi \alpha^{\prime}\left[P^{k}(\tau, 0), P^{j}\left(\tau, \sigma^{\prime}\right)\right] \mathscr{F}_{k}^{i}=-\partial_{\sigma}\left[X^{k}(\tau, \sigma), P^{j}\left(\tau, \sigma^{\prime}\right)\right]_{\sigma=0} M_{k}^{i},
$$

and

$$
2 \pi \alpha^{\prime}\left[P^{k}(\tau, 0), X^{j}\left(\tau, \sigma^{\prime}\right)\right] \mathscr{F}_{k}{ }^{i}=-\partial_{\sigma}\left[X^{i}(\tau, \sigma), X^{j}\left(\tau, \sigma^{\prime}\right)\right]_{\sigma=0} .
$$

These simple relations show that the standard canonical commutation relations for $\mathscr{F}=0$,

$$
\begin{aligned}
& {\left[X^{i}(\tau, \sigma), P_{j}\left(\tau, \sigma^{\prime}\right)\right]=i \delta_{j}^{i} \delta\left(\sigma, \sigma^{\prime}\right),} \\
& {\left[P_{i}(\tau, \sigma), P_{j}\left(\tau, \sigma^{\prime}\right)\right]=0,} \\
& {\left[X^{i}(\tau, \sigma), X^{j}\left(\tau, \sigma^{\prime}\right)\right]=0,}
\end{aligned}
$$

are no longer valid when $\mathscr{F} \neq 0$. They are not compatible with the boundary condition (7) when $\mathscr{F} \neq 0$. In particular, without doing any further calculations, one can see already from (12) that the string coordinates must necessarily be non-commutative somewhere along the string. A consistent quantization was therefore proposed in [6] and it was shown that the canonical commutation relations are modified to

$$
\begin{aligned}
& {\left[P^{i}(\tau, \sigma), P^{j}\left(\tau, \sigma^{\prime}\right)\right]=0,} \\
& {\left[X^{k}(\tau, \sigma), X^{l}\left(\tau, \sigma^{\prime}\right)\right]= \begin{cases} \pm 2 \pi i \alpha^{\prime}\left(M^{-1} \mathscr{F}\right)^{k l}, & \sigma=\sigma^{\prime}=0 \text { or } \pi \\
0, & \text { otherwise, }\end{cases} } \\
& {\left[X^{i}(\tau, \sigma), P^{j}\left(\tau, \sigma^{\prime}\right)\right]=i \eta^{i j} \tilde{\delta}\left(\sigma, \sigma^{\prime}\right),}
\end{aligned}
$$

where $\tilde{\delta}\left(\sigma, \sigma^{\prime}\right)$ is the delta function on $[0, \pi]$ with vanishing derivative at the boundary, e.g. $\partial_{\sigma} \tilde{\delta}\left(\sigma, \sigma^{\prime}\right)=0$ for $\sigma=0, \pi$. See Ref. [6] for its explicit form. Thus we see that the string becomes non-commutative at the endpoint, i.e. the D-brane becomes non-commutative. It was further shown that for the case $\mathscr{F}=B$, (17) agrees with the results obtained from other considerations $[9,10]$. The result of our quantization was later confirmed in [11] using standard string perturbation theory.

Although our quantization in [6] is entirely consistent and produces results in agreement with other considerations, the argument there was mainly based on intuitive guess and consistency argument. It may perhaps be more satisfactory to give a 
"derivation" of that result based on more traditional method. In the next section, we will show that the result in [6] can also be obtained from the standard constraint quantization of Dirac. Consequences of the quantization (16)-(18) in the boundary state formalism (closed string) and D-brane physics are under investigation [12]; as well as the quantization of charged open string [13-15] and its possible consequences ${ }^{1}$.

\section{Dirac quantization of the bosonic sector}

In this section, we compute the Dirac bracket starting from the standard Poisson brackets

$$
\begin{aligned}
& \left(X^{i}(\sigma), P_{j}\left(\sigma^{\prime}\right)\right)=\delta_{j}^{i} \delta\left(\sigma, \sigma^{\prime}\right), \\
& \left(P_{i}(\sigma), P_{j}\left(\sigma^{\prime}\right)\right)=0, \\
& \left(X^{i}(\sigma), X^{j}\left(\sigma^{\prime}\right)\right)=0 .
\end{aligned}
$$

See Refs. $[19,20]$ for a review of the Dirac procedure of constrained quantization. Since

$$
\partial_{\sigma} X^{i}+\partial_{\tau} X^{j} \mathscr{F}_{j}^{i}=2 \pi \alpha^{\prime} P^{j} \mathscr{F}_{j}^{i}+\partial_{\sigma} X^{j} M_{j}^{i},
$$

the boundary condition is a constraint in the phase space

$$
\Phi^{i}(0)=\Phi^{i}(\pi)=0, \quad i=0,1, \ldots, p,
$$

where we have introduced the notation

$$
\Phi^{i}(\sigma)=2 \pi \alpha^{\prime} P^{j} \mathscr{F}_{j}^{i}+\partial_{\sigma} X^{j} M_{j}^{i}
$$

Using the Hamiltonian

$$
H_{B}=\frac{1}{4 \pi \alpha^{\prime}} \int d \sigma\left(\left(\partial_{\tau} X\right)^{2}+\left(\partial_{\sigma} X\right)^{2}\right),
$$

it is easy to show that one requires also the constraints at $\sigma=0, \pi^{2}$

$$
\partial_{\sigma}^{2 \mathrm{n}} \Phi^{i}(\sigma)=0, \quad \partial_{\sigma}^{2 \mathrm{n}+1} P^{i}(\sigma)=0, \quad n=0,1, \ldots,
$$

and that these are all the second class constraints. We will denote them by $\phi^{(\alpha k \mathrm{n})}$, $\alpha=1,2 ; k=0,1, \ldots, p ; n=0,1, \ldots$,

$$
\phi^{(1 k \mathrm{n})}=\partial_{\sigma}^{2 \mathrm{n}} \Phi^{k}, \quad \phi^{(2 k \mathrm{n})}=\partial_{\sigma}^{2 \mathrm{n}+1} P^{k} .
$$

These constraints are consistent with the explicit Fourier mode expansion of the fields $X^{i}$ and $P^{i}$.

\footnotetext{
${ }^{1}$ See in particular the following papers for some recent developments: Ref. [16] studies the closed string interaction in the presence of non-commutative D-branes; the papers [17] and [18] study the effects of the $B$-field in the boundary state formalism.

${ }^{2}$ We thank A. Bilal for a helpful discussion about the issue of equations of motion in determining the secondary constraints.
} 
One can then compute the Poisson matrix $C^{(\alpha k \mathrm{n})(\beta l \mathrm{~m})}$ of the constraints. The basic ones are the $C^{(\alpha k 0)(\beta l 0)}$ ones:

$$
\begin{aligned}
& \left(\Phi^{k}(\sigma), \Phi^{l}\left(\sigma^{\prime}\right)\right)=2 \pi \alpha^{\prime}(\mathscr{F} M)^{k l}\left[\partial_{\sigma} \delta\left(\sigma, \sigma^{\prime}\right)+\partial_{\sigma^{\prime}} \delta\left(\sigma^{\prime}, \sigma\right)\right], \\
& \left(\Phi^{k}(\sigma), \partial_{\sigma^{\prime}} P^{l}\left(\sigma^{\prime}\right)\right)=M^{k l} \partial_{\sigma} \partial_{\sigma^{\prime}} \delta\left(\sigma, \sigma^{\prime}\right), \\
& \left(\partial_{\sigma} P^{k}(\sigma), \partial_{\sigma^{\prime}} P^{l}\left(\sigma^{\prime}\right)\right)=0
\end{aligned}
$$

and in general

$$
C^{(\alpha k \mathrm{n})(\beta l \mathrm{~m})}\left(\sigma, \sigma^{\prime}\right)=\left(\phi^{(\alpha k \mathrm{n})}(\sigma), \phi^{(\beta l \mathrm{~m})}\left(\sigma^{\prime}\right)\right)=\partial_{\sigma}^{2 \mathrm{n}} \partial_{\sigma^{\prime}}^{2 \mathrm{~m}} C^{(\alpha k 0)(\beta l 0)}\left(\sigma, \sigma^{\prime}\right) .
$$

A distinct feature of our case is that the constraints (27) are imposed only at the boundary of the open string. As a result, the Dirac bracket should be given by

$$
\begin{aligned}
\left(A(\sigma), B\left(\sigma^{\prime}\right)\right)^{*}= & \left(A(\sigma), B\left(\sigma^{\prime}\right)\right)-\sum_{\sigma^{\prime \prime} \sigma^{\prime \prime \prime}}\left(A(\sigma), \phi^{(\alpha k \mathrm{n})}\left(\sigma^{\prime \prime}\right)\right) \\
& \times C_{(\alpha k \mathrm{n})(\beta l \mathrm{~m})}\left(\sigma^{\prime \prime}, \sigma^{\prime \prime \prime}\right)\left(\phi^{(\beta l \mathrm{~m})}\left(\sigma^{\prime \prime \prime}\right), B\left(\sigma^{\prime}\right)\right),
\end{aligned}
$$

where in place of an integral, we have a sum $\sigma^{\prime \prime}, \sigma^{\prime \prime \prime}$ over the endpoints $0, \pi$. We also adopt the Einstein summation convention for the indices $k, l, \mathrm{n}, \mathrm{m}$ unless otherwise stated. With an obvious labelling of the columns and rows of the matrix, the inverse $C_{(\alpha k \mathrm{n})(\beta l \mathrm{~m})}\left(\sigma^{\prime \prime}, \sigma^{\prime \prime \prime}\right)$ is given by

$$
\begin{aligned}
& C_{(\alpha k \mathrm{n})(\beta l \mathrm{~m})}\left(\sigma^{\prime \prime}, \sigma^{\prime \prime \prime}\right) \\
& \quad=\left(\begin{array}{cc}
0 & -\left(M^{-1}\right)_{k l} R_{\mathrm{n} \mathrm{m}}\left(\sigma^{\prime \prime}, \sigma^{\prime \prime \prime}\right) \\
\left(M^{-1}\right)_{k l} R_{\mathrm{nm}}\left(\sigma^{\prime \prime}, \sigma^{\prime \prime \prime}\right) & 2 \pi \alpha^{\prime}\left(\mathscr{F} M^{-1}\right)_{k l} S_{\mathrm{n} \mathrm{m}}\left(\sigma^{\prime \prime}, \sigma^{\prime \prime \prime}\right)
\end{array}\right)
\end{aligned}
$$

with $R, S$ satisfying

$$
\begin{aligned}
& \sum_{\sigma^{\prime \prime}} \partial_{\sigma}^{2 \mathrm{n}+1} \partial_{\sigma^{\prime \prime}}^{2 \mathrm{~m}+1} \delta\left(\sigma, \sigma^{\prime \prime}\right) R_{\mathrm{mk}}\left(\sigma^{\prime \prime} \sigma^{\prime \prime \prime}\right)=\delta_{\mathrm{k}}^{\mathrm{n}} \delta_{\sigma \sigma^{\prime \prime \prime}} \\
& \sum_{\sigma^{\prime \prime}} \partial_{\sigma}^{2 \mathrm{n}} \partial_{\sigma^{\prime \prime}}^{2 \mathrm{~m}}\left[\partial_{\sigma} \delta\left(\sigma, \sigma^{\prime \prime}\right)+\partial_{\sigma^{\prime \prime}} \delta\left(\sigma^{\prime \prime}, \sigma\right)\right] R_{\mathrm{mk}}\left(\sigma^{\prime \prime}, \sigma^{\prime \prime \prime}\right) \\
& =\sum_{\sigma^{\prime \prime}} \partial_{\sigma}^{2 \mathrm{n}+1} \partial_{\sigma^{\prime \prime}}^{2 \mathrm{~m}+1} \delta\left(\sigma, \sigma^{\prime \prime}\right) S_{\mathrm{m} \mathrm{k}}\left(\sigma^{\prime \prime}, \sigma^{\prime \prime \prime}\right)
\end{aligned}
$$

It follows immediately from the triangular form of $C_{(\alpha k \mathrm{n})(\beta l \mathrm{~m})}$ that

$$
\left(P^{i}(\sigma), P^{j}\left(\sigma^{\prime}\right)\right)^{*}=0
$$

is not modified. To proceed with the computation of the rest of the Dirac brackets among $X^{i}$ and $P^{i}$, one may try to invert the relations (34), (35) to determine the form of $R$ and $S$ and then use them in (32). However, the explicit form of $R, S$ obtained this way are highly singular and generally contain ambiguities. Fortunately, there is a trick to bypass these steps. We will now show that it is in fact possible to compute the desired brackets using only the defining relations (34) and (35). The detailed form of $R, S$ is not needed. 
We first compute $\left(X^{i}(\sigma), X^{j}\left(\sigma^{\prime}\right)\right)^{*}$, the definition (32) gives

$$
\begin{aligned}
\left(X^{i}(\sigma), X^{j}\left(\sigma^{\prime}\right)\right)^{*}= & -2 \pi \alpha^{\prime}\left(M^{-1} \mathscr{F}\right) \\
& \times \sum_{\sigma^{\prime \prime} \sigma^{\prime \prime \prime}}\left[\partial_{\sigma^{\prime \prime}}^{2 \mathrm{n}} \delta\left(\sigma, \sigma^{\prime \prime}\right) R_{\mathrm{nm}}\left(\sigma^{\prime \prime}, \sigma^{\prime \prime \prime}\right) \partial_{\sigma^{\prime \prime \prime}}^{2 \mathrm{~m}+1} \delta\left(\sigma^{\prime}, \sigma^{\prime \prime \prime}\right)\right. \\
& -\partial_{\sigma^{\prime \prime}}^{2 \mathrm{n}+1} \delta\left(\sigma, \sigma^{\prime \prime}\right) S_{\mathrm{n} \mathrm{m}}\left(\sigma^{\prime \prime}, \sigma^{\prime \prime \prime}\right) \partial_{\sigma^{\prime \prime \prime}}^{2 \mathrm{~m}+1} \delta\left(\sigma^{\prime}, \sigma^{\prime \prime \prime}\right) \\
& \left.+\partial_{\sigma^{\prime \prime}}^{2 \mathrm{n}+1} \delta\left(\sigma, \sigma^{\prime \prime}\right) R_{\mathrm{n} \mathrm{m}}\left(\sigma^{\prime \prime}, \sigma^{\prime \prime \prime}\right) \partial_{\sigma^{\prime \prime \prime}}^{2 \mathrm{~m}} \delta\left(\sigma^{\prime}, \sigma^{\prime \prime \prime}\right)\right] .
\end{aligned}
$$

It is easy to see that $\left(X^{i}(\sigma), X^{j}\left(\sigma^{\prime}\right)\right)^{*}=0$ for $\sigma, \sigma^{\prime}$ not both at the endpoints. Now we multiply (35) for the case $\mathrm{n}=0$ by $\sum_{\mathrm{k}} \sum_{\sigma^{\prime \prime \prime}} \partial_{\sigma^{\prime \prime \prime}}^{2 \mathrm{k}+1} \delta\left(\sigma^{\prime}, \sigma^{\prime \prime \prime}\right)$ and integrate over $\sigma$. We obtain

$$
\begin{aligned}
& \left.\sum_{\sigma^{\prime \prime} \sigma^{\prime \prime \prime}} \partial_{\sigma^{\prime \prime}}^{2 \mathrm{~m}} \delta\left(\sigma, \sigma^{\prime \prime}\right) R_{\mathrm{m} \mathrm{k}}\left(\sigma^{\prime \prime}, \sigma^{\prime \prime \prime}\right) \partial_{\sigma^{\prime \prime \prime}}^{2 \mathrm{k}+1} \delta\left(\sigma^{\prime}, \sigma^{\prime \prime \prime}\right)\right|_{\sigma=0} ^{\sigma=\pi} \\
& \quad=\left.\sum_{\sigma^{\prime \prime} \sigma^{\prime \prime \prime}} \partial_{\sigma^{\prime \prime}}^{2 \mathrm{~m}+1} \delta\left(\sigma, \sigma^{\prime \prime}\right) S_{\mathrm{m} \mathrm{k}}\left(\sigma^{\prime \prime}, \sigma^{\prime \prime \prime}\right) \partial_{\sigma^{\prime \prime \prime}}^{2 \mathrm{k}+1} \delta\left(\sigma^{\prime}, \sigma^{\prime \prime \prime}\right)\right|_{\sigma=0} ^{\sigma=\pi},
\end{aligned}
$$

where we have used $\int d \sigma \partial_{\sigma^{\prime \prime}} \delta\left(\sigma^{\prime \prime}, \sigma\right)=\partial_{\sigma^{\prime \prime}} \int d \sigma \delta\left(\sigma^{\prime \prime}, \sigma\right)=0$ to get rid of the second term on the left-hand side of (35). Therefore the first and second term in (37) cancel each other in $\left(X^{i}(\pi)-X^{i}(0), X^{j}\left(\sigma^{\prime}\right)\right)^{*}$ and we are left with

$$
\begin{aligned}
\left(X^{i}(\pi)-X^{i}(0), X^{j}\left(\sigma^{\prime}\right)\right)^{*}= & -2 \pi \alpha^{\prime}\left(M^{-1} \mathscr{F}\right)^{i j} \sum_{\sigma^{\prime \prime} \sigma^{\prime \prime \prime}} \partial_{\sigma^{\prime \prime}}^{2 \mathrm{n}+1} \delta \\
& \times\left.\left(\sigma, \sigma^{\prime \prime}\right) R_{\mathrm{n} \mathrm{m}}\left(\sigma^{\prime \prime}, \sigma^{\prime \prime \prime}\right) \partial_{\sigma^{\prime \prime \prime}}^{2 \mathrm{~m}} \delta\left(\sigma^{\prime}, \sigma^{\prime \prime \prime}\right)\right|_{\sigma=0} ^{\sigma=\pi} \\
= & -2 \pi \alpha^{\prime}\left(M^{-1} \mathscr{F}\right)^{i j} \cdot\left(\delta_{\sigma^{\prime} 0}+\delta_{\sigma^{\prime} \pi}\right),
\end{aligned}
$$

where we have used in the last step above

$$
\left.\sum_{\sigma^{\prime \prime} \sigma^{\prime \prime \prime}} \partial_{\sigma^{\prime \prime}}^{2 \mathrm{n}+1} \delta\left(\sigma, \sigma^{\prime \prime}\right) R_{\mathrm{nm}}\left(\sigma^{\prime \prime}, \sigma^{\prime \prime \prime}\right) \partial_{\sigma^{\prime \prime \prime}}^{2 \mathrm{~m}} \delta\left(\sigma^{\prime}, \sigma^{\prime \prime \prime}\right)\right|_{\sigma=0} ^{\sigma=\pi}=\delta_{\sigma^{\prime} 0}+\delta_{\sigma^{\prime} \pi} .
$$

This can be obtained by taking the $n=0$ case of (34), multiplying it with $\sum_{\mathrm{k}} \sum_{\sigma^{\prime \prime \prime}} \partial_{\sigma^{\prime \prime \prime}}^{2 \mathrm{k}} \delta\left(\sigma^{\prime}, \sigma^{\prime \prime \prime}\right)$ and integrating over $\sigma$; and note that

$$
\int_{\sigma} \sum_{\sigma^{\prime \prime \prime}} \delta_{\sigma \sigma^{\prime \prime \prime}} \delta\left(\sigma^{\prime}, \sigma^{\prime \prime \prime}\right)=\int_{\sigma} \sum_{\sigma^{\prime \prime \prime}} \delta_{\sigma \sigma^{\prime \prime \prime}} \delta\left(\sigma^{\prime}, \sigma\right)=\sum_{\sigma^{\prime \prime \prime}} \delta_{\sigma^{\prime} \sigma^{\prime \prime \prime}}=\delta_{\sigma^{\prime} 0}+\delta_{\sigma^{\prime} \pi} .
$$

Formally, if one exchange the order of integration and the sum in the above, one gets zero times a delta function. Therefore (41) calls for a better justification. One way to justify it is to use a lattice regularization by replacing the interval $[0, \pi]$ by a lattice of $M$ equidistant points with spacing $\epsilon=\pi / M$,

$$
\delta\left(\sigma, \sigma^{\prime}\right) \rightarrow \frac{1}{\epsilon} \delta_{\sigma \sigma^{\prime}}, \quad \int d \sigma \rightarrow \epsilon \sum_{\sigma} .
$$

Since we know [6] from the D-brane field theory considerations that

$$
\left(X^{i}(0), X^{j}(0)\right)^{*}=-\left(X^{i}(\pi), X^{j}(\pi)\right)^{*},
$$

it follows from (39) that the non-trivial Dirac bracket must be

$$
\left(X^{i}(0), X^{j}(0)\right)^{*}=-\left(X^{i}(\pi), X^{j}(\pi)\right)^{*}=-2 \pi \alpha^{\prime}\left(M^{-1} \mathscr{F}\right)^{i j} .
$$


Next, we compute $\left(X^{i}(\sigma), P^{j}\left(\sigma^{\prime}\right)\right)^{*}$. It is straightforward to obtain

$$
\left(X^{i}(\sigma), P^{j}\left(\sigma^{\prime}\right)\right)^{*}=\eta^{i j}\left(\delta\left(\sigma, \sigma^{\prime}\right)-Q\left(\sigma, \sigma^{\prime}\right)\right),
$$

where

$$
Q\left(\sigma, \sigma^{\prime}\right)=\sum_{\sigma^{\prime \prime} \sigma^{\prime \prime \prime}} \partial_{\sigma^{\prime \prime}}^{2 \mathrm{~m}+1} \delta\left(\sigma, \sigma^{\prime \prime}\right) R_{\mathrm{mk}}\left(\sigma^{\prime \prime}, \sigma^{\prime \prime \prime}\right) \partial_{\sigma^{\prime \prime \prime}}^{2 \mathrm{k}+1} \delta\left(\sigma^{\prime \prime \prime}, \sigma^{\prime}\right) .
$$

Using the definition (34) with $n=0$, it is now easy to show that

$$
\partial_{\sigma} Q\left(\sigma, \sigma^{\prime}\right)=\delta_{\sigma 0} \cdot\left[\partial_{\sigma} \delta\left(\sigma, \sigma^{\prime}\right)\right]_{\sigma=0}+\delta_{\sigma \pi} \cdot\left[\partial_{\sigma} \delta\left(\sigma, \sigma^{\prime}\right)\right]_{\sigma=\pi}
$$

and hence $\tilde{\delta}\left(\sigma, \sigma^{\prime}\right) \equiv \delta\left(\sigma, \sigma^{\prime}\right)-Q\left(\sigma, \sigma^{\prime}\right)$ has the desired property of having a vanishing derivative when one of its arguments is at the boundary; and $\tilde{\delta}\left(\sigma, \sigma^{\prime}\right)$ is just $\delta\left(\sigma, \sigma^{\prime}\right)$ when both arguments are away from the boundary. The Fourier expansion of $\tilde{\delta}\left(\sigma, \sigma^{\prime}\right)$ can be found in [6].

Needless to say, some of the steps presented above are a little formal. The consistent quantization using modes [6] provides a more concrete basis of our calculation here and can be viewed as a complementary viewpoint of the same quantization. From a practical point of view, the modes commutation relations are more useful to perturbative string calculations.

\section{Inclusion of fermions}

In a generic background, the fermionic part of a RNS open string ${ }^{3}$ gets additional couplings, for example

$$
\frac{-i}{4 \pi \alpha^{\prime}} \int B_{i j} \bar{\psi}^{i} \epsilon^{\alpha \beta} \rho_{\alpha} \partial_{\beta} \psi^{j}
$$

The situation is much simpler for our case with the flat background (2) and the complete supersymmetric Lagrangian can be easily written down. The equation of motion is not modified

$$
\bar{\partial} \psi_{+}^{i}=\partial \psi_{-}^{i}=0,
$$

where $\partial=\partial_{\tau}+\partial_{\sigma}, \bar{\partial}=\partial_{\tau}-\partial_{\sigma}$. The open string world-sheet in the type IIand type 0 string theories has the supersymmetry

$$
\delta X^{\mu}=\bar{\epsilon} \psi^{\mu}, \quad \delta \psi^{\mu}=-i \rho^{\alpha} \partial_{\alpha} X^{\mu} \epsilon
$$

with the preserved supersymmetry parametrized by

$$
\epsilon_{+}=\lambda \epsilon_{-}, \quad \lambda= \pm 1 .
$$

\footnotetext{
${ }^{3} \mathrm{We}$ use the convention of two-dimensional spinor algebra in [21]. In particular, $\psi=\left(\begin{array}{l}\psi_{+} \\ \psi_{-}\end{array}\right), \rho^{0}=\left(\begin{array}{cc}0 & 1 \\ -1 & 0\end{array}\right), \rho^{1}=\left(\begin{array}{ll}0 & 1 \\ 1 & 0\end{array}\right)$
} 
The supersymmetric boundary condition compatible with (7) is ${ }^{4}$

$$
\begin{aligned}
& \psi_{+}^{i}(\eta+\mathscr{F})_{i}^{j}+\lambda \psi_{-}^{i}(\eta-\mathscr{F})_{i}^{j}=0, \quad i, j=0,1, \ldots, p, \\
& \psi_{+}^{a}+\lambda \psi_{-}^{a}=0, \quad a=p+1, \ldots, 9,
\end{aligned}
$$

for $\sigma=0, \pi$.

The constrained quantization on the fermions can be similarly performed as in the bosonic case above. However, since we know that $\psi^{i}$ and $X^{i}$ are related by supersymmetry and we have already derived the commutation relations for the $X^{i}$, s, it is perhaps more interesting not to repeat the Dirac quantization here, but instead to utilize the power of world-sheet supersymmetry to derive the Dirac bracket for the $\psi^{i}$, s directly. This approach is expected to be particularly useful in more complicated cases. For example in the case [22] where the background is not constant but weak enough so that one can still determine the leading non-trivial commutation relation for the open string; and in the case of supersymmetric $\mathrm{AdS}_{k} \times S^{k}$ background [12] relevant for the AdS/CFT correspondance.

It is easy to see that $\left(\psi^{\mu}(\sigma), X^{\nu}\left(\sigma^{\prime}\right)\right)^{*}=0$. The reason is that the original Poisson bracket $\left(\psi^{\mu}(\sigma), X^{\nu}\left(\sigma^{\prime}\right)\right)$ is zero and the constraints never mix the bosonic and fermionic fields, so this Poisson bracket is not modified by the Dirac procedure. Applying (50), we obtain

$$
0=\delta\left(\psi^{\mu}, X^{\nu}\right)^{*}=\left(\begin{array}{c}
{\left[i\left(\partial X^{\mu}, X^{\nu}\right)^{*}+\left(\psi_{+}^{\mu}, \psi_{+}^{\nu}\right)^{*}-\lambda\left(\psi_{+}^{\mu}, \psi_{-}^{\nu}\right)^{*}\right] \cdot \epsilon_{-}} \\
\cdot \lambda \epsilon_{-}
\end{array}\right)
$$

Now we will concentrate on the modified boundary condition (52). The Dirac bracket relations for the tranverse directions can be obtained simply by setting $\mathscr{F}=0$ in the following. Substituting (52) into the above, we obtain

$$
\begin{aligned}
\left(\psi_{+}^{i}, \psi_{+}^{j}\right)^{*} & =\frac{-i}{2}\left(\partial X^{i}, X^{k}\right)^{*}(\eta-\mathscr{F})_{k}{ }^{j}, \\
\left(\psi_{-}^{i}, \psi_{-}^{j}\right)^{*} & =\frac{-i}{2}\left(\bar{\partial} X^{i}, X^{k}\right)^{*}(\eta+\mathscr{F})_{k}{ }^{j} .
\end{aligned}
$$

At $\sigma=0, \pi$, the use of (9) allows one to express $\partial_{\sigma} X^{i}$ and $\partial_{\tau} X^{i}$ in terms of $P^{k}$

$$
\partial_{\sigma} X^{i}=-2 \pi \alpha^{\prime} P^{k}\left(\mathscr{F} M^{-1}\right)_{k}{ }^{i}, \quad \partial_{\tau} X^{i}=2 \pi \alpha^{\prime} P^{k}\left(M^{-1}\right)_{k}{ }^{i} .
$$

Thus

$$
\begin{aligned}
& \left(\partial X^{i}(\sigma), X^{j}\left(\sigma^{\prime}\right)\right)^{*}=2 \pi \alpha^{\prime}\left(M^{-1}(\eta+\mathscr{F})\right)^{i j} \tilde{\delta}\left(\sigma, \sigma^{\prime}\right), \\
& \left(\bar{\partial} X^{i}(\sigma), X^{j}\left(\sigma^{\prime}\right)\right)^{*}=2 \pi \alpha^{\prime}\left(M^{-1}(\eta-\mathscr{F})\right)^{i j} \tilde{\delta}\left(\sigma, \sigma^{\prime}\right),
\end{aligned}
$$

and so (55) implies

$$
\left(\psi_{+}^{i}(\sigma), \psi_{+}^{j}\left(\sigma^{\prime}\right)\right)^{*}=\left(\psi_{-}^{i}(\sigma), \psi_{-}^{j}\left(\sigma^{\prime}\right)\right)^{*}=-i \pi \alpha^{\prime} \eta^{i j} \tilde{\delta}\left(\sigma, \sigma^{\prime}\right),
$$

\footnotetext{
${ }^{4}$ We thank V. Schomerus for a useful email exchange about this boundary condition. A different boundary condition was used in [6] which corresponds to a non-supersymmetric D-brane.
} 
with $\sigma$ and $\sigma^{\prime}$ at the boundary. It is also easy to see that in the interior of the string, the Dirac bracket is not modified and takes the same form as (59). Therefore the fermion commutator is not modified (except for the modification of the delta function) by the presence of $\mathscr{F}$ and takes the standard form.

\section{Remarks}

In the quantization procedure of Dirac, Eq. (41) is the only ambiguity. But as mentioned in Section 2, it is inconsistent to say that (41) vanishes. Also note that the ambiguity resides only in the commutation relations for the endpoint coordinates, the quantization for the space-time coordinates in the interior of the opens string has no ambiguity, and their commutation relations are the same as if $\mathscr{F}=0$. The lattice regularization we choose there gives the same result we obtained in [6]. It leads to the conclusion that a D-brane in the $B$ field background has a non-commutative worldvolume in the sense that its world-volume theory is the SYM theory living on a non-commutative space.

Upon quantization, the Dirac bracket becomes the (anti)commutator of the operators. From the point of view of the open string, the modifications to the commutation relations is "not very much". But as discussed in [6], since the endpoint of the open string is living on the D-brane, the D-brane world-volume becomes a non-commutative one. It is well known that a non-commutative manifold can be described in the dual language of the algebra of functions living on it, one immediately arrives at the conclusion that the D-brane world-volume field theory is a non-commutative one, i.e. one with a modified multiplication. In fact it is easy to check that our modified commutators (44) are equivalent to the following Moyal product [23,24] defined on the D-brane world-volume [1],

$$
f(\xi) \star g\left(\xi^{\prime}\right)=\left.\exp \left(\Theta^{i j} \frac{\partial}{\partial \xi^{i}} \frac{\partial}{\partial \xi^{\prime j}}\right) f(\xi) g\left(\xi^{\prime}\right)\right|_{\xi=\xi^{\prime}},
$$

where $\xi^{i}, i=0, \ldots, p$ are the D-brane world-volume coordinates and

$$
\Theta^{i j}= \pm i \pi \alpha^{\prime}\left(\mathscr{F} M^{-1}\right)^{i j} \text {. }
$$

The non-commutative $\mathrm{D} p$-brane world-volume theory takes functionally the same form as the $\mathrm{SYM}_{9+1}$ theory dimensionally reduced to $p+1$ dimensions, with the usual product of fields replaced by the Moyal product (60), and with the space-time integral replaced by a cyclic trace. It has been argued [5] that in the DLCQ limit of $\epsilon \rightarrow 0$ with

$$
\begin{array}{ll}
X^{\mu} \sim \epsilon, & B \sim 1 / \epsilon^{2}, \\
\alpha^{\prime} \sim \epsilon, & g_{s} \sim \epsilon^{3 / 2},
\end{array}
$$

where $g_{s}$ is the string coupling constant, the SYM Lagrangian is exact and hence deserves attention.

When this work is nearly finished, we learned that another group [25] has also employed Dirac's procedure for the same problem, but their final result is different from ours. The reason is presumably that they chose a different regularization. 


\section{Acknowledgements}

We thank A. Bilal, M. Cederwall, Y.-C. Kao, D. Matalliotakis, R. Russo and V. Schomerus for helpful discussions. The work of C.S.C. is supported by the Swiss National Science Foundation. The work of P.M.H. is supported in part by the National Science Council, Taiwan, R.O.C.

\section{References}

[1] A. Connes, M.R. Douglas, A. Schwarz, J. High Energy Phys. 02 (1998) 003. hep-th/9711162.

[2] N. Seiberg, Phys. Rev. Lett. 79 (1997) 3577. hep-th/9710009.

[3] M.R. Douglas, C. Hull, J. High Energy Phys. 2 (1998) 8. hep-th/9711165.

[4] C. Hofman, E. Verlinde, U-duality of Born-Infeld on the non-commutative two-torus, hep-th/9810116.

[5] N. Seiberg, talk given at the Conference New Ideas in Particle Physics and Cosmology, Univ. Penn., May 19-22, 99; transpancies available at http://dept.physics.upenn.edu/particle_meeting/seiberg.html.

[6] C.-S. Chu, P.-M. Ho, Nucl. Phys. B 550 (1999) 151. hep-th/9812219.

[7] J. Dai, R.G. Leigh, J. Polchinski, Mod. Phys. Lett. A 4 (1989) 2073.

[8] R. Leigh, Mod. Phys. Lett. A 4 (1989) 2767.

[9] Y.-K.E. Cheung, M. Krogh, Nucl. Phys. B 528 (1998) 185.

[10] T. Kawano, K. Okuyama, Phys. Lett. B 433 (1998) 29.

[11] V. Schomerus, D-branes and deformation quantization, hep-th/9903205.

[12] C.-S. Chu, P.-M. Ho, work in progress.

[13] A. Abouelsaood, C.G. Callan, C.R. Nappi, S.A. Yost, Nucl. Phys. B 280 (1987) 599.

[14] C. Bachas, Phys. Lett. B 296 (1992) 77. hep-th/9209032.

[15] C. Bachas, Phys. Lett. B 374 (1996) 37. hep-th/9511043.

[16] S. Hyun, Y. Kiem, S. Lee, C.-Y. Lee, Closed string interacting with non-commutative D-branes, hep-th/9909059.

[17] N. Ishibashi, A relation between commutative and non-commutative descriptions of D-branes, hepth/9909176.

[18] K. Okuyama, A path integral representation of the map between commutative and non-commutative gauge fields, hep-th/9910138.

[19] P.A.M. Dirac, Lectures on Quantum Mechanics, Yeshiva University, 1964.

[20] A. Hanson, T. Regge, C. Teitelboim, Constrained Hamiltonian Systems, RX-748, 1976. Contribution to the Lincei Interdisciplinary Centre for Mathematical Sciences and their Applications, no. 22.

[21] D. Lúst, S. Theisen, Lectures on String Theory (Springer, Berlin, 1989).

[22] C.-S. Chu, P.-M. Ho, Y.-C. Kao, World-volume uncertainty relations for D-branes, hep-th/9904133.

[23] J.E. Moyal, Proc. Camb. Phil. Soc. 45 (1949) 99.

[24] F. Bayen, M. Flato, C. Fronsdal, A. Lichnerowicz, D. Sternheimer, Ann. Phys. 111 (1978) 61.

[25] F. Ardalan, H. Arfaei, M.M. Sheikh-Jabbari, Dirac quantization of open strings and non-commutativity in branes, hep-th/9906161. 